antibodies to parvovirus B19 results were further confirmed by Western blot.

Results In 28 of 50 patients with RA (56\%) and in 7/26 of the controls (26.9\%) IgG antibodies to parvovirus B19 were detected $(\mathrm{x} 2, \mathrm{p}<0.05)$. In 9 of 24 patients with OA and in $7 /$ 26 of controls IgG antibodies to parvovirus B19 were detected (x2, p > 0.05). In 11 of 50 patients with RA (22\%) IgM antibodies to parvovirus B19 were detected and the results were further confirmed by Western blot, while in none of the patients with $\mathrm{OA}$ and in none of the controls were IgM antibodies observed (x2, p $<0.001$ and $\mathrm{p}>0.05$, respectively).

Conclusion The prevalence of antibodies to parvovirus B19 was found to be increased in patients with RA as compared to controls. In patients with OA the prevalence of antibodies to parvovirus B19 was found to be comparable to that in controls. These results indicate either that infection with parvovirus B19 may be implicated in the pathogenesis of RA, as previously discussed in the literature, or that some cases of chronic polyarthropathy due to parvovirus B19 may have been misdiagnosed as rheumatoid arthritis.

\section{THU0109 THE DESTRUCTIVE PROCESS OF RHEUMATOID ARTHRITIS SYNOVIAL FIBROBLASTS (RA-SF) IS INDEPENDENT OF P53}

${ }^{1} \mathrm{CA}$ Seemayer, ${ }^{1} \mathrm{~S}$ Kuchen, ${ }^{1} \mathrm{M}$ Neihart, ${ }^{1} \mathrm{P}$ Kuenzler, ${ }^{2} \mathrm{E}$ Neumann, ${ }^{3} \mathrm{M}$ Pruschy, ${ }^{1} \mathrm{~T}$ Pap, ${ }^{4}$ WK Aicher, ${ }^{1} \mathrm{U}$ Muller-Ladner, ${ }^{1} \mathrm{BA}$ Michel, ${ }^{1} \mathrm{RE}$ Gay, ${ }^{1} \mathrm{~S}$ Gay. ${ }^{1}$ Center Exp Rheumat; ${ }^{2}$ Int Medicine, University, Regensburg; ${ }^{3}$ Rad Onc, University Hospital, Zürich, Switzerland; ${ }^{4} B a s$ Sci Lab, Orth Surg, Tübingen, Germany

10.1136/annrheumdis-2001.986

\section{Background}

Objectives The aim of this study was to investigate, whether the destructive process of synovial fibroblasts in rheumatoid arthritis is dependent on the expression of p53. Moreover, we searched for the expression of p53 in RA-SF in vitro and in vivo. In addition, we examined the inducibility of p53 in RA-SF by X-rays in vitro.

Methods Paraffin embedded synovial tissues from 14 RA, 3 osteoarthritis (OA), 3 normal synovia and a neurological p53 positive tumour were investigated with DO7 anti-p53 antibodies by immunohistochemistry. RA-SF from passages 2 to 12 ( $\mathrm{n}=$ 10), OA-SF $(\mathrm{n}=2)$, normal synoviocytes $(\mathrm{N}-\mathrm{SF}, \mathrm{n}=1)$ and foreskin fibroblasts (FSFB, $\mathrm{n}=1)$ ) from passages 4-8 were grown on chamber slides and analysed with the same primary antibodies utilising immunofluorescence. SV40 transformed RASF (HSE) and SV40 transformed N-SF (K4IM) as well as the SV40 transformed bronchial epithelial cell line BEAS 2B served as positive controls. The percentage of positive cells was evaluated by a scoring system. RA-SF and HSE cultured in vitro were co-implanted with human cartilage under the renal capsule or the under the skin of SCID mice and kept there for 60 days. Paraffin sections from these SCID mice experiments were stained for $\mathrm{p} 53$. In addition, sections from former SCID mouse experiments including retroviral and adenoviral gene transfer studies revealing a strong invasion of synovial cells into the cartilage were investigated for the expression of $\mathrm{p} 53$. Moreover, RA-SF ( $\mathrm{n}$ $=2$ ) and controls (FSFB, $\mathrm{n}=1$ ) were irradiated with 10 Gy (Xrays) and analysed time dependently for the expression of p53 by immunofluorescence.

Results In vivo, p53 was not abundantly expressed in RA synovial tissue and in particular not at sites of invasion. In general, less than $5 \%$ of the cells in RA and OA synovial tissues stained positively for $\mathrm{p} 53$, while no staining in the normal synovia occurred. In contrast, the positive control revealed a strong nuclear p53 signal in almost $100 \%$ of the tumour cells. In vitro, the expression rate of p53 in RA-SF was comparable low, while the SV40 transformed cells demonstrated a strong and specific nuclear staining. Furthermore, RA-SF invading the human cartilage in the SCID mouse co-implantation model did not show any expression of p53 at sites of invasion, whereas the implanted SV40 transformed HSE revealed a strong p53 staining indicating that those cells survived at least for 60 days in SCID mice. RA$\mathrm{SF}$ and FSFB cultured in vitro responded equally to the irradiation with X-rays by producing transiently $\mathrm{p} 53$, demonstrating an adequate and functional response of $\mathrm{p} 53$.

Conclusion We suggest that the expression of p53 plays only a limited role in the pathogenesis of RA and particularly not in the invasive process of RA-SF.

\section{THU0110 V-ATPASE INHIBITOR BAFILOMYCIN A1 REDUCES PROTON SECRETION IN FIBROBLASTS}

${ }^{1}$ B Stein, ${ }^{2} \mathrm{~T}$ Pap, ${ }^{1} \mathrm{M}$ George, ${ }^{1} \mathrm{WJ}$ Parak, ${ }^{3} \mathrm{O}$ Müller, ${ }^{2} \mathrm{~S}$ Gay, ${ }^{3} \mathrm{WK}$ Aicher. ${ }^{1} / n s t i t u t e$ for Applied Physics, LMU München, Munich; ${ }^{2}$ WHO Rheumatology Res. Centre, University Hospital, Zurich, Switzerland; ${ }^{3}$ Centre Orthopedic Surgery, University Medical School, Tübingen, Germany

\subsection{6/annrheumdis-2001.987}

Background Metabolic activation of synovial fibroblasts $\left(\mathrm{SF},{ }^{1}\right)$, prosthesis loosening fibroblasts $\left(\mathrm{PLF}^{2}\right)$ and osteoblasts $\left(\mathrm{OB},{ }^{3}\right)$ results in an enhanced acid secretion as determined by cytosensor microphysiometer. In addition, PLF were shown to degrade bone in absence of infiltrating macrophages or osteoclasts.

Objectives We were interested to study if the proton secretion was associated with the activity of specific proton pump, especially with the activity of V-type $\mathrm{H} \pm$ ATPases.

Methods Fibroblasts or osteoblasts were expanded in complete medium containing 10\% FCS and antibiotics. For cytosensor analysis cells were seeded at 30000 cells $/ \mathrm{ml}$ in microcups, mounted into the cytosensor. For metabolic activation the medium was enriched with cytokines such as TNF-alpha, IL-1 (Roche) or different agents such as ionomycin or PMA (Calbiochem) at different concentrations. To block V-ATPase activity, cells were incubated with Bafilomycin A1 or Amiloride (Calbiochem).

Results Metabolic activation as determined by enhanced acid secretion was observed upon stimulation of the fibroblasts with TNF-alpha, IL-1, PDGF, ionomycin or PMA but not with IL-6 or bFGF. Osteoblasts were activated by TNF-alpha, ionomycin and PMA; bFGF, IL-1, IL-6 or PDGF are not tested yet. Acid secretion occurred as early as $20-30 \mathrm{~min}$. after initial stimulation. Under identical conditions, immortalised synovial fibroblast clones secreted less acid when compared to normal fibroblasts. As metabolic activation stimulates glycolysis, the acidification may be simply a consequence of carbohydrate catabolism generating acetate, lactate or carbonate. Therefore fibroblasts were incubated in medium containing Bafilomycin A1, a specific Vtype $\mathrm{H} \pm$ ATPase blocker. Bafilomycin reduced the proton secretion in a time course experiment within $20 \mathrm{~min}$. irreversibly. Interestingly, Amiloride inhibited the proton secretion reversibly. Conclusion Metabolic activation of fibroblasts or osteoblasts by pro-inflammatory cytokines results in enhanced acid secretion. As pro-inflammatory cytokines enhance the expression of matrix degrading proteases as well, we conclude that the acidification of the pericellular milieu by mesenchymal cells accelerates matrix 
degradation. In addition, we provide for the first time experimental evidence that the acid secreted upon metabolic activation is not only a product of anaerobe (lactate, acetate) or aerobe (carbonate) glycolysis, but may be associated with a specific VATPase located in vacuolar membranes in the cytoplasm or on the surface of the cells. Preliminary data corroborate this conclusion, since a V-ATPase was located on the cell surface by immunohistochemistry and a V-ATPase encoding mRNA was detected by $\mathrm{RT} / \mathrm{PCR}$ in the respective cells.

\section{REFERENCES}

1 Parak, et al. Metabolic activation stimulates acid production in synovial fibroblasts. J Rheumatol. 2000;27:2312

2 Sainsbury, et al. Cathepsin K expression by activated fibroblasts at the bone interface of the pseudosynovium in aseptic prosthesis loosening. Proc. ORS Conv. Anaheim \# 27, 1999

3 Mast A. Charakterisierung von Knochenzellen mit biophysikalischen Methoden auf zellulärem Niveau, Master Thesis, Fakult. of Physics, University of Tübingen, 1999

\section{THU0111 FC GAMMA RECEPTOR I AND III EXPRESSION BY MACROPHAGES OF COLLAGEN TYPE II SUSCEPTIBLE MICE IS HIGHER AND DIFFERENTLY REGULATED COMPARED TO OTHER STRAINS}

${ }^{1} \mathrm{AB}$ Blom, ${ }^{1} \mathrm{PL}$ Van Lent, ${ }^{1} \mathrm{AE}$ Holthuysen, ${ }^{2} \mathrm{C}$ Jacobs, ${ }^{3}$ I Tchetverikov, ${ }^{1} \mathrm{LB}$ Van de Putte, ${ }^{1}$ WB Van den Berg. 'Rheumatology; ${ }^{2}$ Nephrology, University Medical Centre St. Radboud, Nijmegen; ${ }^{3}$ TNO Prevention and Health, Leiden, The Netherlands

\subsection{6/annrheumdis-2001.988}

Background In a previous study we found that knee joints of mice, susceptible for collagen type II arthritis (DBA/1, B10. RIII) display enhanced sensitivity to immune complexes (ICs) compared to other strains $(\mathrm{C} 57 \mathrm{BL} / 6, \mathrm{BALB} / \mathrm{c})$. Higher and prolonged inflammation and more severe cartilage damage was observed. Murine synovial macrophages as well as IL-1 determine IC mediated arthritis and these cells communicate with ICs using three classes of $\mathrm{Fc} \gamma$ receptors mediating intracellular signalling: Fc $\gamma \mathrm{RI}$ and III are activating and FcyRII is an inhibitory receptor.

Objectives To investigate whether macrophages of mice that are prone to develop collagen type II arthritis are hypersensitive to ICs by differential Fc $\gamma \mathrm{R}$ expression and regulation.

Methods Basal expression of Fc $\gamma \mathrm{R}$ on macrophages of all strains was determined by immunohistochemistry on naïve whole knee joint sections and FACS-analysis using 2.4G2 antibody. Peritoneal macrophages were stimulated with ICs and cells were processed for RT-PCR. Using primers specific for Fc $\gamma$ RI, II or III semiquantitative RT-PCR was performed. In addition, peritoneal macrophages of all strains and of mice lacking functional Fc $\gamma \mathrm{RI}$ and III (FCR $\gamma$-chain ${ }^{-/}$) were stimulated and tested for the production of IL-1 and stromelysin.

Results Basal expression of Fc $\gamma \mathrm{R}$ was significantly higher on synovial and peritoneal macrophages of DBA/1 and B10. RIII mice (mean fluorescence resp $440 \pm 50$ and $360 \pm 30$ ) if compared to $\mathrm{C} 57 \mathrm{BL} / 6$ and $\mathrm{BALB} / \mathrm{c}$ (mean fluorescence resp $240 \pm$ 30 and $280 \pm 30$ ). After stimulation with ICs, mRNA levels of $\mathrm{Fc} \gamma \mathrm{RI}$ and III were significantly higher in DBA/1 and B10. RIII mice. At day 2, Fc $\gamma R I$ expression was $50 \times$ higher and Fc $\gamma$ RIII was $10 \times$ higher. At day 3, Fc $\gamma R$ I and III expression were both $10 \times$ higher. In contrast, Fc $\gamma$ RII mRNA levels were down regulated $50 \times$ for $\mathrm{DBA} / 1$ and $10 \times$ for $\mathrm{B} 10$. RIII if compared to $\mathrm{C} 57 \mathrm{BL} / 6$ and $\mathrm{BALB} / \mathrm{c}$. When DBA/1 and B10RIII peritoneal macrophages were stimulated with ICs $(100 \mu \mathrm{g} / \mathrm{ml})$, a twofold higher and prolonged production of IL-1 was measured if compared to other strains. Macrophages of $\mathrm{FcR} \gamma$-chain ${ }^{-/-}$mice showed no detectable amounts of IL-1 after IC stimulation, indicating the role of FcyR in stimulation of cells using ICs. In addition, also stromelysin production was significantly increased in DBA/1 macrophages.

Conclusion Collagen type II arthritis sensitive mice are hyper reactive to IC through higher basal expression of Fc $\gamma R$ on macrophages of these strains and stronger up regulation of stimulatory as well as down regulation of inhibitory Fc $\gamma$ Receptors, resulting in higher and prolonged expression of IL-1 and stromelysin.

\section{THU0112 ANAEMIA IN RHEUMATOID ARTHRITIS}

${ }^{1} \mathrm{P}$ Athanassiou, ${ }^{2} \mathrm{E}$ Griva, ${ }^{3} \mathrm{I}$ Kostoglou-Athanassiou, ${ }^{1} \mathrm{P}$ Konstantopoulou, ${ }^{4} \mathrm{~S}$ Papazoglou, ${ }^{5} \mathrm{D}$ Rontogianni, ${ }^{1} \mathrm{G}$ Vezyroglou, ${ }^{4} \mathrm{~T}$ Kontomerkos. ${ }^{1}$ Department of Rheumatology, Asklepieion Hospital, Voula; ${ }^{2}$ Department of Haematology; ${ }^{3}$ Department of Endocrinology, Metaxa Hospital, Pireaus, Greece; ${ }^{4}$ Department of Rheumatology; ${ }^{5}$ Department of Histopathology, General Hospital of Athens "G. Gennimatas", Athens

\subsection{6/annrheumdis-2001.989}

Background Rheumatoid arthritis (RA) is accompanied by haematological abnormalities. It is well known that anaemia in RA may have plural aetiology, however no correlation has been performed between anaemia and the morphology of blood cell precursors in the bone marrow in RA.

Objectives The aim was to investigate the aetiology of anaemia in RA by analysing blood, bone marrow aspirate and bone marrow biopsy findings.

Methods RA patients were included in the study, $\mathrm{n}=51$, aged $58.7 \pm 1.2$ (range $17-85$ ) years. None of the patients was taking any disease modifying agents and 26 were newly diagnosed and had never taken any treatment for RA, duration of disease of the remaining being $9.1 \pm 1.8$ (range $0.5-35$ ) years. In all patients antinuclear antibodies, anti-dsDNA antibodies and serum rheumatoid factor were measured. Haematocrit, haemoglobin, MCV, $\mathrm{MCH}, \mathrm{MCHC}$, serum iron, serum ferritin, serum iron-binding capacity, serum folate and vitamin B12 levels were also measured and indirect and direct Coombs' test was performed. In all patients a bone marrow aspirate was obtained and a bone marrow biopsy was performed.

Results In 51 RA patients haemoglobin levels were $11.3+0.2 \mathrm{~g} /$ $\mathrm{dl}($ mean \pm SEM), haematocrit $35.0 \pm 0.6 \%$, MCV $80.5 \pm 1.4$ fl, $\mathrm{MCH} 25.9 \pm 0.5 \mathrm{pg}, \mathrm{MCHC} 32.0 \pm 0.1 \%$, serum iron levels $72.9 \pm 4.8 ? \mathrm{~g} / \mathrm{dl}$, serum iron-binding capacity $343.4 \pm 7.4 \mathrm{?g} / \mathrm{dl}$, ferritin levels $119.5 \pm 23.1 \mathrm{?g} / \mathrm{dl}$, folate $12.4 \pm 1.9 \mathrm{ng} / \mathrm{ml}$ and vitamin B12 levels $722.6 \pm 301.3 \mathrm{pg} / \mathrm{ml}$. In 32 of 51 patients with haemoglobin $12 \mathrm{~g} / \mathrm{dl}$, hyperplasia of red blood cell precursors was noted in 12, megaloblastoid alterations in 4 and erythroblastic islets in 2 patients. No correlation was observed between the presence or absence of anaemia and the dysplastic alterations in the blood cell precursors in the bone marrow in the RA patients studied.

Conclusion The aetiology of anaemia in RA patients seems to be multifactorial. Dysplastic alterations in blood cell precursors were noted in all the groups of RA patients, irrespective of the presence of anaemia, indicating either that inflammatory cytokines may affect the microenvironment of the bone marrow in RA or that in RA the stem cell in the bone marrow is defective being implicated in the pathogenesis of the disease. 